\title{
Detection and Correction of Doppler Biases in Kalman Filter-based Positioning
}

\author{
Atsushi Mouri ${ }^{1,2}$, Yukihiro Kubo ${ }^{2}$, and Sueo Sugimoto ${ }^{2}$ \\ ${ }^{1}$ Sanda works, Mitsubishi Electric Corporation \\ 2-3-33, Miwa, Sanda-city, Hyogo 669-1513 Japan \\ Tel: +81-(0)79-559-3841, Fax: +81-(0)79-559-3875 \\ ${ }^{2}$ Department of Electrical and Electronic Engineering, Ritsumeikan University \\ Noji-Higashi, Kusatsu City, Shiga 525-8577 Japan \\ Tel: +81-(0)77-561-5972, Fax: +81-(0)77-561-2663 \\ E-mail; Mori.Atsushi@dc.MitsubishiElectric.co.jp
}

\begin{abstract}
In this paper, we present methods of detecting and correcting Doppler biases which cause positioning errors in Doppler-aided Kalman filter-based GNSS (Global Navigation Satellite System) positioning. It is well known that Doppler frequency shift observables are more precise compared with $\mathrm{C} / \mathrm{A}$ code pseudorange observables. Doppler shifts are transformed into range-rates and utilized to smooth code pseudoranges. And Doppler shift observables are immune to cycleslip and continuously available as long as the receiver can track the signals. The precise raw Doppler shift observables can be further exploited for RTK (Real Time Kinematic) positioning to provide robust carrierphase anomaly detection and correction processes $[5,6]$. Therefore Doppler shift observables can be utilized on a priority basis in Doppler-aided Kalman filter-based positioning than $\mathrm{C} / \mathrm{A}$ code pseudoranges. However unexpected Doppler bias errors cause position errors or route departures in the positioning. If the Doppler shifts include bias errors, they can be detected by monitoring the innovation processes in Kalman filter. And then they can be corrected by the exclusion and estimation methods.
\end{abstract}

\section{Introduction}

In this paper, we present methods of detecting and correcting Doppler biases which cause positioning errors in Doppler-aided Kalman filter-based GNSS (Global Navigation Satellite System) positioning.

We have already developed PPP algorithms based on GR(GNSS Regression) models and Kalman filtering for RTK-GNSS positioning [1, 2]. Our derived RTK precise point positioning algorithm achieved the positioning accuracy in decimeter level. However in a downtown street or an urban canyon, multipath signals are dominant than direct signals and hence pseudoranges will be severely degraded. And precise carrier-phase observables are either missing or contaminated with cycle-slips and other observable anomalies. In order to maintain high precision positioning and navigation with GNSS in the severe situation, the algorithms of detecting the cycle slips and multipath based on the statistical test of innovation processes in Kalman filtering are applied for the positioning $[3,4]$.

After detecting the cycle slips and multipath, few available normal GNSS observables can be utilized to get the integer ambiguities. In order to compensate this, Doppler frequency shift observables which a receiver can generate together can be utilized for RTKPPP algorithm. Doppler shift observables are typically available continuously as long as the receiver can track the signals, and are immune to cycle-slips and multipath $[5,6,7]$.

We preliminarily analyze Doppler frequency shift accuracy compared with L1-C/A code pseudoranges. The pseudorange observables are badly affected by the indirect waves under multipath environments, on the other hand Doppler shifts are not almost affected by them. Therefore pseudoranges smoothed by Doppler observables can be utilized for the positioning $[5,6]$. However when Doppler shift observables would have bias errors, Doppler dominant positioning causes positioning errors even under open sky environments.

We actually had positioning errors caused by the Doppler biases of SBAS (WAAS) satellite in real test drives. The Doppler bias is extracted from the satellite signal as a simulation sample. Appropriate noise variances for L1-C/A code pseudoranges and Doppler frequency shifts are set for Kalman Filter-based positioning simulation [8]. We simulate the positioning error caused by the Doppler bias under open sky environment.

The statistical tests based on the innovation processes of the Kalman filtering can be applied for detecting anomalous observables. The $\chi^{2}$ tests based on the normalized innovation processes can be applied for detecting cycle-slips or multipath [9]. We apply the method 
to the detection of Doppler biases on the signals broadcasted from SBAS satellites. Although the $\chi^{2}$ test detection is done at the single observation epoch, the proposed method can be easily extended to the detection based on multiple epochs data.

Furthermore we develop two methods to remove Doppler bias impacts, which are Doppler bias exclusion or Doppler bias estimation. When the Doppler biases are detected by the $\chi^{2}$ tests, the impacts of them are individually removed by each of the methods, and the positioning errors are corrected.

Finally correction results of the two methods are shown and discussed for the feasibility of our methods presented in this paper for detecting Doppler biases and correcting them in Kalman Filter-based positioning.

\section{GR models for Doppler-based Kalman Filter positioning}

Based on [7], we show a simplified GR model which contains the $\mathrm{C} / \mathrm{A}$ code pseudorange and Doppler frequency shift observables. The natural extensions of GNSS regression models for multiple frequencies of Galileo and US-GPS modernization are also similarly formulated.

We consider the following fundamental observables of the pseudoranges $\rho_{C A, u}^{p}(t)$ based on $\mathrm{C} / \mathrm{A}$ codes, as follows:

$$
\begin{aligned}
\rho_{C A, u}^{p}(t)= & r_{u}^{p}\left(t, t-\tau_{u}^{p}\right)+\delta I_{u}^{p}(t)+\delta T_{u}^{p}(t) \\
& +c\left[\delta t_{u}(t)-\delta t^{p}\left(t-\tau_{u}^{p}\right)\right] \\
& +\delta b_{C A, u}-\delta b_{C A}^{p}+e_{C A, u}^{p}(t)
\end{aligned}
$$

where $c\left(\approx 2.99792458 \times 10^{8}[\mathrm{~m} / \mathrm{s}]\right)$ denotes the speed of light, and $f_{1}$ and $\lambda_{1}$ are the central frequency and the wave length of the L1 carrier wave

$$
f_{1}=2 \times 77 \times 10.23[\mathrm{MHz}]=1575.42[\mathrm{MHz}]
$$

In (1), the so-called receiver's biases, $\left\{\delta b_{C A, u}\right\}$, and the satellite biases, $\left\{\delta b_{C A}^{p}\right\}$, are contained in the usual observed positioning data consisting of L1 pseudoranges based on $\mathrm{C} / \mathrm{A}$ codes. Also $r_{u}^{p}\left(t, t-\tau_{u}^{p}\right)$ is the geometric distance between the receiver $u$ at the time $t$ and the satellite $p$ at the time $t-\tau_{u}^{p}\left(\tau_{u}^{p}\right.$ denotes the travel time from the satellite $p\left(p=1, \ldots, n_{s}\right)$ to the receiver $\left.u\right)$. Namely,

$$
\begin{aligned}
r_{u}^{p}(t) \equiv & r_{u}^{p}\left(t, t-\tau_{u}^{p}\right) \\
= & {\left[\left(x_{u}(t)-x^{p}\left(t-\tau_{u}^{p}\right)\right)^{2}+\left(y_{u}(t)-y^{p}\left(t-\tau_{u}^{p}\right)\right)^{2}\right.} \\
& \left.+\left(z_{u}(t)-z^{p}\left(t-\tau_{u}^{p}\right)\right)^{2}\right]^{1 / 2} \\
= & \left\|u(t)-s^{p}\left(t-\tau_{u}^{p}\right)\right\|,
\end{aligned}
$$

where $u \equiv\left[x_{u}, y_{u}, z_{u}\right]^{\mathrm{T}}$ and $s^{p} \equiv\left[x^{p}, y^{p}, z^{p}\right]^{\mathrm{T}}$ are the user (unknown) and satellite positions, respectively.
Also $n_{s}$ shows the number of the observable satellites. Further in (1), $\delta I_{u}^{p}(t)$ and $\delta T_{u}^{p}(t)$ reflect the delay or the advance associated with the transmission of the L1 signal through the ionosphere and the troposphere, respectively. $\delta t_{u}(t)$ and $\delta t^{p}\left(t-\tau_{u}^{p}\right)$ are the clock errors of the receiver $u$ at the time $t$ and the satellite $p$ at the time $t-\tau_{u}^{p}$. $N_{u}^{p}$ denotes integer ambiguity between the satellite $p$ and the receiver $u$, and $\varepsilon^{p}(t), e^{p}(t)$ denote observation errors.

Eq. (3) contains the satellite orbital errors. The estimated satellite orbits are obtained from the navigation messages which are decoded from the transmitted L1 signal. Let us denote $\hat{s}$ as the estimated position of the satellite $s$ at the time $t-\tau_{u}^{p}$.

We use the following relations of the derivatives

$$
\begin{array}{ll}
\frac{\partial r_{u}^{p}}{\partial x_{u}}=\frac{\left(x_{u}-x^{p}\right)}{r_{u}^{p}}, & \frac{\partial r_{u}^{p}}{\partial y_{u}}=\frac{\left(y_{u}-y^{p}\right)}{r_{u}^{p}}, \\
\frac{\partial r_{u}^{p}}{\partial z_{u}}=\frac{\left(z_{u}-z^{p}\right)}{r_{u}^{p}}, & \left(p=1,2, \ldots, n_{s}\right),
\end{array}
$$

and

$$
\begin{array}{ll}
\frac{\partial r_{u}^{p}}{\partial x^{p}}=-\frac{\left(x_{u}-x^{p}\right)}{r_{u}^{p}}, & \frac{\partial r_{u}^{p}}{\partial y^{p}}=-\frac{\left(y_{u}-y^{p}\right)}{r_{u}^{p}} \\
\frac{\partial r_{u}^{p}}{\partial z^{p}}=-\frac{\left(z_{u}-z^{p}\right)}{r_{u}^{p}}, & \left(p=1,2, \ldots, n_{s}\right) .
\end{array}
$$

Then we have the relation:

$$
\frac{\partial r_{u}^{p}}{\partial u}=-\frac{\partial r_{u}^{p}}{\partial s^{p}}
$$

Thus the 1st order Taylor series approximation of (3) around the previous estimated value $u=\hat{u}^{(j)}$ and $s^{p}=$ $\hat{s}^{p}$ is given by

$$
\begin{aligned}
r_{u}^{p} & \cong r_{\hat{u}^{(j)}}^{\hat{p}}+g_{\hat{u}^{(j)}}^{\hat{p}}\left[u-s^{p}-\left(\hat{u}^{(j)}-\hat{s}^{p}\right)\right] \\
& =\left\|\hat{u}^{(j)}-\hat{s}^{p}\right\|+\frac{\left(\hat{u}^{(j)}-\hat{s}^{p}\right)^{\mathrm{T}}}{\left\|\hat{u}^{(j)}-\hat{s}^{p}\right\|}\left[u-s^{p}-\left(\hat{u}^{(j)}-\hat{s}^{p}\right)\right] \\
& =\frac{\left(\hat{u}^{(j)}-\hat{s}^{p}\right)^{\mathrm{T}}}{\left\|\hat{u}^{(j)}-\hat{s}^{p}\right\|}\left(u-s^{p}\right)
\end{aligned}
$$

for $p=1,2, \ldots, n_{s}$, where

$$
g_{\hat{u}^{(j)}}^{\hat{p}} \equiv\left[\frac{\partial r_{u}^{p}}{\partial u}\right]_{u=\hat{u}^{(j)}, s^{p}=\hat{s}^{p}}^{\mathrm{T}}=\frac{\left(\hat{u}^{(j)}-\hat{s}^{p}\right)^{\mathrm{T}}}{\left\|\hat{u}^{(j)}-\hat{s}^{p}\right\|}
$$

From (1), therefore, we have the approximation:

$$
\begin{aligned}
\rho_{C A, u}^{p} \cong & g_{\hat{u}^{(j)}}^{\hat{p}}\left(u-s^{p}\right) \\
& +\delta I_{u}^{p}+\delta T_{u}^{p}+c\left(\delta t_{u}-\delta t^{p}\right) \\
& +\delta b_{C A, u}-\delta b_{C A}^{p}+e_{C A, u}^{p},
\end{aligned}
$$

Define the $n_{s} \times 3$ matrix:

$$
G_{\hat{u}^{(j)}}^{\hat{s}} \equiv\left[\begin{array}{c}
g_{\hat{u}^{(j)}}^{\hat{1}} \\
g_{\hat{u}^{(j)}}^{2} \\
\vdots \\
g_{\hat{u}_{s}^{(j)}}^{\hat{n}_{s}}
\end{array}\right],
$$


namely,

$$
G_{\hat{u}^{(j)}}^{\hat{s}}=\left[\begin{array}{ccc}
\frac{\partial r_{\hat{u}^{(j)}}^{\hat{1}^{(j)}}}{\partial \hat{x}_{u}^{(j)}} & \frac{\partial r_{\hat{u}^{(j)}}^{\hat{1}}}{\partial \hat{y}_{u}^{(j)}} & \frac{\partial r_{\hat{u}^{(j)}}^{\hat{1}}}{\partial \hat{z}_{u}^{(j)}} \\
\frac{\partial r_{\hat{u}^{(j)}}^{\hat{2}}}{\partial \hat{x}_{u}^{(j)}} & \frac{\partial r_{\hat{u}^{(j)}}^{\hat{2}}}{\partial \hat{y}_{u}^{(j)}} & \frac{\partial r_{\hat{u}^{(j)}}^{\hat{2}}}{\partial \hat{z}_{u}^{(j)}} \\
\vdots & \vdots & \vdots \\
\frac{\partial r_{\hat{u}^{(j)}}^{\hat{n}_{s}}}{\partial \hat{x}_{u}^{(j)}} & \frac{\partial r_{\hat{u}^{(j)}}^{\hat{n}_{s}}}{\partial \hat{y}_{u}^{(j)}} & \frac{\partial r_{\hat{u}^{(j)}}^{\hat{n}_{s}}}{\partial \hat{z}_{u}^{(j)}}
\end{array}\right] .
$$

Now let us discuss the satellite's as well as receiver's hardware delays $\delta b^{p}$, and $\delta b_{*, u}$. It was pointed out in that the magnitude of the satellite's hardware delay bias is usually in the range of (several nanosecond $\times c$ ) while the receiver's hardware biases could exceed (10 nanoseconds $\times c$ ). Therefore, we assume that the satellite's hardware delay biases may be negligible. On the other hand, we do not disregard the receiver's hardware delay such that we define the terms the $1 \times 1$ vector

$$
\delta b_{u} \equiv\left[\delta b_{C A, u}\right]^{\mathrm{T}} .
$$

However, in this paper, we assume that $\delta b_{u}$ is known constant such that the GNSS observable data $\rho_{C A, u}^{p}$ are compensated.

We have approximately the Doppler shift data $D_{L 1, u}^{p}$ for L1 carrier frequency, as follows:

$$
\begin{aligned}
& \lambda_{1} D_{L 1, u}^{p} \\
\cong & g_{\hat{u}^{(j)}}^{\hat{p}}\left(\dot{u}-\dot{s}^{p}\right)+c \dot{\delta} t_{u}+\lambda_{1} \varepsilon_{D L 1, u}^{p},
\end{aligned}
$$

And the receiver's clock errors are generally modeled,

$$
\begin{aligned}
& c \delta t_{u, t+1}=c \delta t_{u, t}+\Delta_{t} c \dot{\delta} t_{u, t}+w_{c \delta t_{u}, t} \\
& c \dot{\delta} t_{u, t+1}=c \dot{\delta} t_{u, t}+w_{c \dot{\delta} t_{u}, t},
\end{aligned}
$$

where $\Delta_{t}$ denotes the sampling interval of the receiver's clock error, and $w_{c \delta t_{u}, t}$ and $w_{c \dot{\delta} t_{u}, t}$ are assumed as Gaussian white noise processes with zero means and covariances $q_{c \delta t}$ and $q_{c \dot{\delta} t}$, respectively. Then we can write

$$
\begin{aligned}
{\left[\begin{array}{c}
c \delta t_{u, t+1} \\
c \dot{\delta} t_{u, t+1}
\end{array}\right] } & =\left[\begin{array}{cc}
1 & \Delta_{t} \\
0 & 1
\end{array}\right]\left[\begin{array}{c}
c \delta t_{u, t} \\
c \dot{\delta} t_{u, t}
\end{array}\right]+\left[\begin{array}{l}
w_{c \delta t_{u}, t} \\
w_{c \dot{\delta} t_{u}, t}
\end{array}\right] \\
& \equiv F_{A, c \delta t}\left[\begin{array}{c}
c \delta t_{u, t} \\
c \dot{\delta} t_{u, t}
\end{array}\right]+\left[\begin{array}{l}
w_{c \delta t_{u}, t} \\
w_{c \dot{\delta} t_{u}, t}
\end{array}\right]
\end{aligned}
$$

Here, we define the vectors:

$$
\begin{aligned}
\rho_{C A, u}^{s} \equiv\left[\begin{array}{c}
\rho_{C A, u}^{1} \\
\vdots \\
\rho_{C A, u}^{n_{s}}
\end{array}\right], \quad D_{L 1, u}^{s} \equiv\left[\begin{array}{c}
D_{L 1, u}^{1} \\
\vdots \\
D_{L 1, u}^{n_{s}}
\end{array}\right], \\
c \delta t^{s} \equiv\left[\begin{array}{c}
c \delta t^{1} \\
\vdots \\
c \delta t^{n_{s}}
\end{array}\right], \\
s \equiv\left[\begin{array}{c}
s^{1} \\
\vdots \\
s^{n_{s}}
\end{array}\right], \quad \dot{s} \equiv\left[\begin{array}{c}
\dot{s}^{1} \\
\vdots \\
\dot{s}^{n_{s}}
\end{array}\right], \\
\delta I_{u}^{1} \\
\equiv\left[\begin{array}{c}
\delta T_{u}^{1} \\
\vdots \\
\delta I_{u}^{n_{s}}
\end{array}\right], \quad \delta T_{u} \equiv\left[\begin{array}{c}
\delta T_{u}^{n_{s}} \\
e_{C A, u}^{1} \\
\vdots \\
e_{C A, u}^{n_{s}}
\end{array}\right], \quad \varepsilon_{D L 1, u} \equiv\left[\begin{array}{c}
\varepsilon_{D L 1, u}^{1} \\
\vdots \\
\varepsilon_{D L 1, u}^{n_{s}}
\end{array}\right],
\end{aligned}
$$

Furthermore, from (11), we define a block diagonal matrix with the size $\left(n_{s} \times 3 n_{s}\right)$ :

$$
G_{D, \hat{u}^{(j)}}^{\hat{s}} \equiv\left[\begin{array}{ccccc}
g_{\hat{u}^{(j)}}^{\hat{1}} & O & O & \cdots & O \\
O & g_{\hat{u}^{(j)}}^{2} & O & \cdots & O \\
\vdots & & \ddots & & \vdots \\
\vdots & & & \ddots & O \\
O & \cdots & \cdots & O & g_{\hat{u}^{(j)}}^{\hat{n}_{s}}
\end{array}\right] .
$$

Then from (9)-(12), we have the following vector regression equation:

$$
y_{u}^{s}=H_{\hat{u}^{(j)}}^{\hat{s}} \theta_{u}+v_{u},
$$

where

$$
\begin{aligned}
& y_{u}^{s} \equiv\left[\begin{array}{c}
\rho_{C A, u}^{s} \\
\lambda_{1} D_{L 1, u}^{s}
\end{array}\right], \quad \theta_{u} \equiv\left[\begin{array}{c}
u \\
\dot{u} \\
c \delta t_{u} \\
c \dot{\delta} t_{u} \\
c \delta t^{s} \\
s \\
\dot{s} \\
\delta I_{u} \\
\delta T_{u}
\end{array}\right]
\end{aligned}
$$

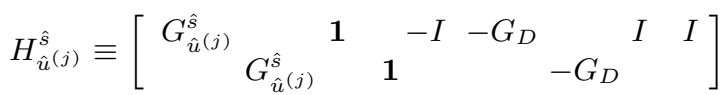

$$
\begin{aligned}
& v_{u} \equiv\left[e_{C A, u}^{\mathrm{T}}, \lambda_{1}\left(\varepsilon_{D L 1, u}^{p}\right)^{\mathrm{T}}\right]^{\mathrm{T}},
\end{aligned}
$$


and $I$ denote the $n_{s} \times n_{s}$ identity matrix and $\mathbf{1} \equiv$ $[1,1, \cdots, 1]^{\mathrm{T}}: n_{s} \times 1$ vector .

Now we assume that the information of the satellite position $s$, the time derivative of the satellite position $\dot{s}$, the satellite clock error $c \delta t^{s}$ as well as the delay or the advance due to the ionospheric and tropospheric effects, $\delta I_{u}^{\mathrm{T}}$ and $\delta T_{u}^{\mathrm{T}}$ are provided with uncertainties as follows

$$
\begin{aligned}
\hat{s} & =s+e_{s}, \\
\hat{\dot{s}} & =\dot{s}+e_{\dot{s}}, \\
c \hat{\delta} t^{s} & =c \delta t+e_{\delta t^{s}},
\end{aligned}
$$

and

$$
\begin{gathered}
\hat{\delta I_{u}}=\delta I_{u}+e_{\delta I_{u}}, \\
\hat{\delta T_{u}}=\delta T_{u}+e_{\delta T_{u}},
\end{gathered}
$$

where $e_{s}, \cdots, e_{\delta T_{u}}$ are white Gaussian noises denoting uncertainty of the information.

From (22)-(26), we have

$$
\begin{aligned}
s & =\hat{s}-e_{s}, \\
\dot{s} & =\hat{\dot{s}}-e_{\dot{s}}, \\
c \delta t^{p} & =c \hat{\delta} t^{s}-e_{\delta t^{s}}, \\
\delta I_{u} & =\hat{\delta I_{u}}-e_{\delta I_{u}}, \\
\delta T_{u} & =\delta \hat{T}_{u}-e_{\delta T_{u}} .
\end{aligned}
$$

Substituting the above equations (27)-(31) into the GR equation (18), we have

$$
y_{u} \equiv\left[\begin{array}{c}
y_{C A, \hat{u}^{(j)}}^{\hat{s}} \\
y_{D L 1, \hat{u}^{(j)}}^{\hat{s}}
\end{array}\right]=C_{\hat{u}^{(j)}}^{\hat{s}^{\prime}}\left[\begin{array}{c}
u \\
\dot{u} \\
c \delta t_{u} \\
c \dot{\delta} t_{u}
\end{array}\right]+v
$$

where

$$
\begin{aligned}
& y_{C A, \hat{u}^{(j)}}^{\hat{s}} \equiv \rho_{C A, u}^{s}+G_{D, \hat{u}^{(j)}}^{\hat{s}} \hat{s} \\
& +c \hat{\delta} t^{s}-\hat{\delta I_{u}}-\hat{\delta T_{u}}, \\
& y_{D L 1, \hat{u}^{(j)}}^{\hat{s}} \equiv \lambda_{2} D_{L 1, u}^{s}+G_{D, \hat{u}^{(j)}}^{\hat{s}} \hat{\dot{s}} \text {, } \\
& C_{\hat{u}^{(j)}}^{\hat{s}}=\left[\begin{array}{llll}
G_{\hat{u}^{(j)}}^{\hat{s}} & & \mathbf{1} & \\
& G_{\hat{u}^{(j)}}^{\hat{s}} & & \mathbf{1}
\end{array}\right],
\end{aligned}
$$

and

$$
v=\left[\begin{array}{c}
G_{D, \hat{u}^{(j)}}^{\hat{s}} e_{s}+e_{\delta t^{s}}-e_{\delta I_{u}}-e_{\delta T_{u}}+e_{C A, u} \\
G_{D, \hat{u}^{(j)}}^{\hat{s}} e_{\dot{s}}+\lambda_{1} \varepsilon_{D L 1, u}^{p}
\end{array}\right] .
$$

\section{Kalman Filter formulation}

Let us derive the Kalman filtering algorithm for positioning based upon the GR equation in (32). For this purpose, we derive the so-called state equation as follows.

\section{STATE EQUATIONS}

Let us derive the state equation for kinematic positioning. Since the kinematic case requires mathematical models of the moving objects, we often apply one of the dynamical models which are assumed as first-order Markov processes of, the velocity of $u(: v)$, of the acceleration of $u(: a)$ (the so-called Singer's moving model [10]), or of the jerk of $u(: \gamma),[11,12]$, with or without the constraints $[13,14]$.

For the land vehicle such as automobiles, we adopt Singer's model for the east-west (E) coordinate and the north-south $(\mathrm{N})$ coordinate, and a first order markov model of the velocity for the up-down (U) coordinate, in the local frame [2]. Namely, the unknowns are the position, velocity, and horizontal accelerations in ENU coordinate frame. Therefore the state vector is defined as follows:

$$
\eta \equiv\left[u^{\mathrm{T}}, \dot{u}^{\mathrm{T}}, c \delta t_{u}, c \dot{\delta} t_{u}\right]^{\mathrm{T}}
$$

we define the state vector as follows:

$$
\begin{aligned}
\eta_{L} \equiv & {\left[u_{L}^{\mathrm{T}}, \dot{u}_{L}^{\mathrm{T}}, a_{L}^{\mathrm{T}}, c \delta t_{u}, c \dot{\delta} t_{u}\right]^{\mathrm{T}} } \\
\equiv & {\left[x_{L}, y_{L}, z_{L}, v_{x, L}, v_{y, L}, v_{z, L}, a_{x, L}, a_{y, L}\right.} \\
& \left.\quad c \delta t_{u}, c \dot{\delta} t_{u}\right]^{\mathrm{T}}
\end{aligned}
$$

where $L$ stands for the coordinates in the local frame (or the local-level system (LLS), or east-north-up (ENU) system) $[2,15]$. Then the transformation matrix to ENU coordinates from WGS- 84 coordinates is given by

$$
T_{W}^{L}=\left[\begin{array}{ccc}
-\sin \lambda & \cos \lambda & 0 \\
-\sin \phi \cos \lambda & -\sin \phi \sin \lambda & \cos \phi \\
\cos \phi \cos \lambda & \cos \phi \sin \lambda & \sin \phi
\end{array}\right]
$$

where $\lambda$ and $\phi$ denote the longitude and latitude, respectively. Let $u_{0}$ in the WGS-84 coordinate system be an origin in the ENU coordinate system, then any coordinate $u$ in the WGS- 84 system can be described by the ENU system by the relation:

$$
u_{L}=T_{W}^{L}\left(u-u_{0}\right)
$$

Therefore, also we have

$$
u=u_{0}+\left(T_{W}^{L}\right)^{-1} u_{L} .
$$

Furthermore by the relation $\left(T_{W}^{L}\right)^{-1}=\left(T_{W}^{L}\right)^{\mathrm{T}}$, we have

$$
\begin{aligned}
u \equiv\left[\begin{array}{l}
x \\
y \\
z
\end{array}\right] & =\left[\begin{array}{l}
x_{0} \\
y_{0} \\
z_{0}
\end{array}\right]+\left(T_{W}^{L}\right)^{\mathrm{T}}\left[\begin{array}{l}
x_{L} \\
y_{L} \\
z_{L}
\end{array}\right] \\
& =u_{0}+\left(T_{W}^{L}\right)^{T} u_{L},
\end{aligned}
$$

where

$$
u_{L} \equiv\left[x_{L}, y_{L}, z_{L}\right]^{\mathrm{T}} .
$$


Then by the assumption of the Singer's model, namely the accelerations; $a_{x, L}, a_{y, L}$, of $x_{L}, y_{L}$, respectively, are assumed as the first order Markov processes:

$$
\begin{aligned}
& \dot{a}_{x_{L}}(t)=-\alpha_{x} a_{x_{L}}(t)+w_{a_{x}}(t) \\
& \dot{a}_{y_{L}}(t)=-\alpha_{y} a_{y_{L}}(t)+w_{a_{y}}(t) .
\end{aligned}
$$

Also the velocity of $v_{z, L}$ of $z_{L}$ is assumed as a first order Markov process:

$$
\dot{v}_{z_{L}}(t)=-\alpha_{z} v_{z_{L}}(t)+w_{v_{z}}(t)
$$

Then, we have the following form of the discrete-time state equation [2]:

$$
\eta_{L}(t+1)=A(t) \eta_{L}(t)+B(t) w(t)
$$

and the observation equation is also obtained from the relation between (32) and (41) as follows:

$$
\begin{gathered}
y(t)=C(t) \eta_{L}(t)+v(t) \\
C=\left[\begin{array}{cccc}
G_{\hat{u}^{(j)}}^{\hat{s}} T_{W}^{L} & & & \\
& G_{\hat{u}^{(j)}}^{\hat{s}} T_{W}^{L} & & \mathbf{1}
\end{array}\right]
\end{gathered}
$$

The positioning algorithm based on the Kalman filter for (45) and (46) can be summarized as follows [1, 2]:

$$
\begin{aligned}
& \hat{\eta}_{L}(t+1 \mid t)=A(t) \hat{\eta}_{L}(t \mid t) \\
& P(t+1 \mid t)=A(t) P(t \mid t) A^{\mathrm{T}}(t)+Q(t) \\
& \text { (: Predicted step) } \\
& \nu(t) \equiv y(t)-C(t) \hat{\eta}_{L}(t \mid t-1) \\
& \text { (: Innovation process) } \\
& \hat{\eta}_{L}(t \mid t)=\hat{\eta}_{L}(t \mid t-1)+K(t) \nu(t) \\
& P(t \mid t)=P(t \mid t-1) \\
& -K(t) C(t) P(t \mid t-1) \\
& \text { (: Update step) } \\
& K(t)=P(t \mid t-1) C^{\mathrm{T}}(t) \\
& {\left[C(t) \times P(t \mid t-1) C^{\mathrm{T}}(t)+R(t)\right]^{-1}} \\
& \text { (: Kalman gain) } \\
& \text { Init. Cond.: } \quad\left\{\begin{array}{l}
\hat{\eta}_{L}(0 \mid-1)=\bar{\eta}_{L}(0) \\
P(0 \mid-1)=P(0)
\end{array}\right.
\end{aligned}
$$

where $Q(t)$ is covariance matrix of system noise, and $R(t)$ is covariance matrix of observation noise.

\section{Preliminary analysis}

\subsection{Doppler bias sample}

We analyze Doppler frequency shift accuracy compared with L1-C/A code pseudoranges. Fig.1 shows the comparison between the delta-ranges derived from code pseudoranges and the range-rates derived from Doppler shifts. The $\mathrm{C} / \mathrm{A}$ code delta-ranges are the timedifferenced pseudorange observables in (1) over two

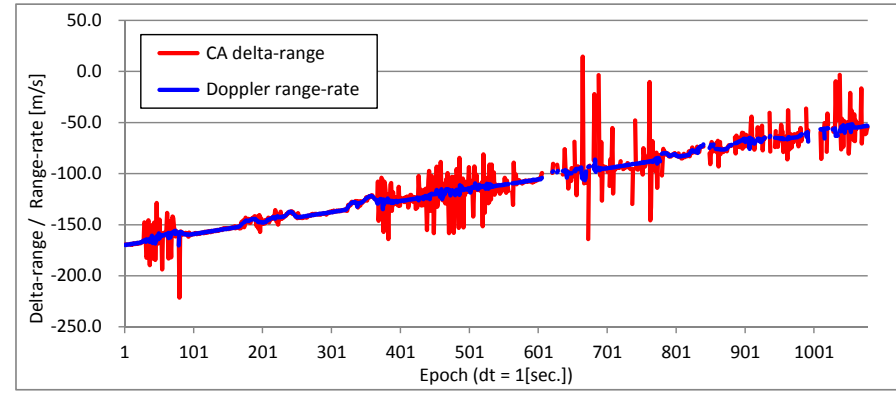

Fig. 1: CA code delta-range / Doppler shift range-rate

succesive epochs, namely $\rho_{C A, u}(t)-\rho_{C A, u}(t-1)$. On the other hand, Doppler shift range-rates are receiversatellite values based on Doppler frequency shift observables, namely $\lambda D_{L 1, u}(t)$ in (12). The observables can be collected from the satellite whose elevation is $55-56$ degrees under urban canyon environments. The deltaranges have several tens of meter fluctuations, on the other hand the range-rates do not almost have fluctuations. The pseudorange observables are badly affected by the indirect waves under multipath environments, on the other hand Doppler shifts are not almost affected by those. Therefore Doppler observables have the potential to be utilized to smooth the code pseudorange noises in GNSS difficult environment. In practice, a method of Kalman filter-based Doppler-smoothing of code pseudorange are reported $[5,6]$. Doppler dominant positioning causes positioning errors even under open sky environments, if Doppler shift observables would have bias errors. We had a long time positioning error in real test drives, and extract the Doppler bias from SBAS(WAAS) observables. In the test drives, the GPS and SBAS data of the automobile were obtained by the receiver $(: \mathrm{u}$-blox $\mathrm{NEO}-7 \mathrm{~N}$ receiver, measurable only one frequency: L1), with the antenna equipped

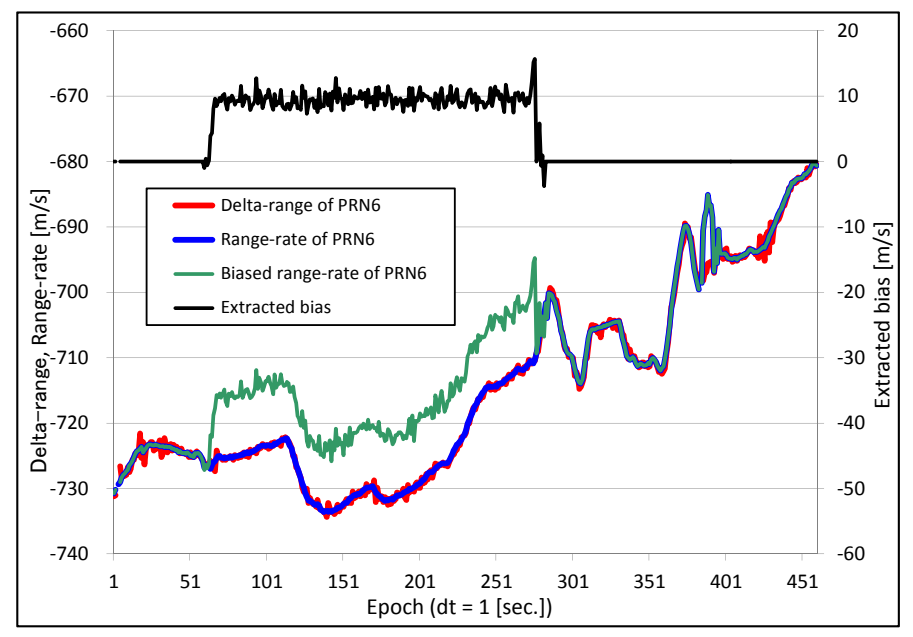

Fig. 2: Simulation bias sample 
in the test vehicle, when the automobile moved in the streets in Maryland, US, on April 15, 2014, from 17:14' 41 to $17: 32$ ' 05 UTC. The Doppler range-rate bias (see Fig. 2) is extracted by subtracting the delta-ranges from the range-rates of SBAS signal, and has the rectangular shape whose length is from epoch 60 to 280 and size is about $10[\mathrm{~m} / \mathrm{s}]$. The extracted Doppler rangerate bias is added to the original range-rate of PRN6 as a simulation sample for GPS based positioning. The delta-ranges of PRN6 are almost the same values as the original range-rates of PRN6 because of open sky environment. And the biased range-rate of PRN6 has synchronously almost the same amount of bias as the extracted bias.

\subsection{Simulating the positioning error}

The analysis of Kalman filter-based positioning has been carried out to simulate the positioning error. The $\mathrm{C} / \mathrm{A}$ code pseudoranges and Doppler shift observables which include the biased range-rate of PRN6 are prepared from the real test drive log, and used for the simulation.

Generally speaking, standard error model says that one-sigma error of pseudoranges is about $5[\mathrm{~m}]$ [8]. As Doppler shifts are more accurate, one-sigma error of the shifts is supposed to be about one tenth of pseudorange errors that is about $0.5[\mathrm{~m} / \mathrm{s}]$. We have the Kalman filter positioning with $5[\mathrm{~m}]$ and $0.5[\mathrm{~m} / \mathrm{s}]$ as typical positioning for $\mathrm{C} / \mathrm{A}$ code and Doppler shift observables, respectively (see Table 1 ). And for comparison we use two other different simulation conditions as C/A based positioning or Doppler based positioning.

Table 1: Noise variance for $\mathrm{R}(\mathrm{t})$

\begin{tabular}{|l||c|c|}
\hline Noise variance & $\begin{array}{c}\text { C/A } \\
\text { code } \\
\text { pseudoranges } \\
{\left[\mathrm{m}^{2}\right]}\end{array}$ & $\begin{array}{c}\text { Doppler } \\
\text { frequency } \\
\text { shifts } \\
{\left[(\mathrm{m} / \mathrm{s})^{2}\right]}\end{array}$ \\
\hline C/A based positioning & $0.3^{2}$ & $3^{2}$ \\
\hline Typical positioning & $5.0^{2}$ & $0.5^{2}$ \\
\hline Doppler based positioning & $10.0^{2}$ & $0.3^{2}$ \\
\hline
\end{tabular}

As for typical positioning, the positioning errors from the vehicle position is gradually getting bigger according to the vehicle movement. The continuous Doppler bias causes abnormal velocity vector, and then the continuous abnormal velocity vector finally causes the bigger positioning errors. In the case of Doppler based positioning with bigger $\mathrm{C} / \mathrm{A}$ noise and less Doppler noise variance, the amount of positioning errors are the biggest among three cases. As for C/A based positioning, the positioning errors are smaller than other two cases, although the biased Doppler is used for the positioning (see Fig. 3). The $\mathrm{C} / \mathrm{A}$ pseudoranges are utilized for the positioning on a priority basis.

Fig. 4 shows the ENU errors of simulation results. The positioning results by original log data which include no Doppler bias are regarded as the reference trajectory for

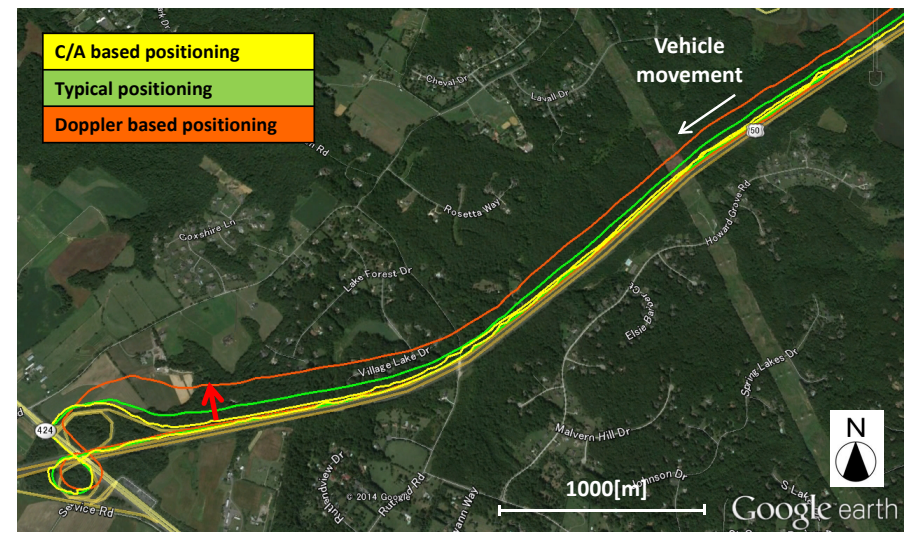

Fig. 3: Simulation results

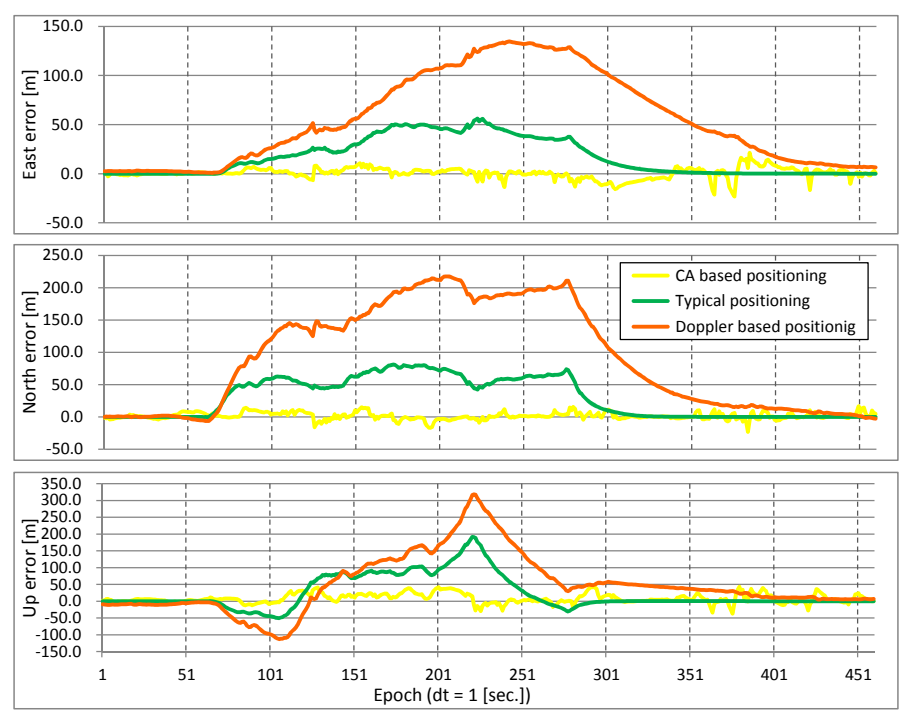

Fig. 4: ENU errors of simulation

evaluating positioning errors. In the case of typical positioning, the positioning error is simulated even under open sky environment, and has about $50[\mathrm{~m}]$ error to the direction of east and north. Doppler based positioning is badly affected by the bias, and causes the errors after epoch 280 which includes no Doppler bias.

\section{Detection and correction of Doppler bias}

\subsection{Innovation process detection}

Since the innovation process in (50) is a white Gaussian with 0 mean and covariance matrix $M(t)(=$ $\left.\left[M_{i j}(t)\right] ; i, j=1, \cdots, 2 n_{s}\right)$ under the hypothesis $H_{0}$ such that there are no Doppler biases. $M(t)$ is formulated as follows [4].

$$
\begin{aligned}
M(t) & \equiv \mathrm{E}\left[\nu(t) \nu^{\mathrm{T}}(t)\right] \\
& =\left[C(t) P(t \mid t-1) C^{\mathrm{T}}(t)+R(t)\right]
\end{aligned}
$$

where $P(t \mid t-1)$ is the error covariance matrix of $\eta_{L}(t \mid t-1)$. Therefore, under the hypothesis $H_{0}$, the 
$j$-th element of innovation vector $\nu$ which is expressed by $\nu_{j}$ would be the normal distribution with 0 mean and variance $M_{j j}$. Then $\nu_{j}$ can be normalized as follows.

$$
\begin{aligned}
& \nu_{n, j}(t) \equiv \sqrt{\frac{1}{M_{j j}(t)}} \nu_{j}(t), \quad j=1, \ldots, 2 n_{s} \\
& \mathrm{E}\left[\nu_{n, j}(t)\right]=0, \quad \operatorname{Var}\left[\nu_{n, j}(t)\right]=1
\end{aligned}
$$

Based on the equation (55), the anomaly of Doppler frequency observables can be detected by monitoring the normalized innovation processes corresponding to the Doppler shift observables, i.e. $\nu_{n, j}$ for $j=n_{s}+$ $1, \ldots, 2 n_{s}$. Each element of $\nu_{n, j}(t)$ is statistically independent to other elements, and each is a Gaussian process. Therefore the statistics $T_{j}(t)$ of the squared value of $\nu_{n, j}(t)$ follows the $\chi^{2}$ distribution with 1 degree of freedom, namely,

$$
T_{j}(t)=\left(\nu_{n, j}(t)\right)^{2}
$$

To effectively detect the Doppler anomaly, we focus on $\chi^{2}$ test of each Doppler shift observable. If Doppler biases occur, the covariance matrix of the innovation process changes. Therefore we formulate two hypotheses such as,

\section{$H_{0}$ : the anomal bias does not occur \\ $H_{1}$ : the anomal bias occurs}

Our decision rule of accept or reject the hypothesis is as follows; If $T_{j}(t)$ is larger than the upper percent point $\chi_{\alpha}^{2}(1)$ where $\alpha$ is upper probability $(\alpha=0.10,0.05$ etc. $)$ and 1 degree of freedom, then the observed data of Doppler shift contain a bias (accept $H_{1}$ ).

\subsection{Correction of Doppler bias}

The biased Doppler shift observables are detected by the innovation processes, and the bias impacts are removed by the following two methods which are Doppler bias exclusion, or Doppler bias estimation.

In the case of Doppler bias exclusion, if the Doppler bias is detected in the $j$-th satellite, then the related observable, i.e. the $j$-th component of $y_{D L 1 \hat{u}}^{\hat{s}}$ and the related row of observation matrix are excluded. And the observation noises related to $y_{D L 1, \hat{u}}^{\hat{s}}$ are similarly excluded from the covariance matrix $R(t)$ in (53).

For example, if $j=2$, the $2_{n d}$ Doppler observable and the related row components with brackets are excluded as follows.

$$
\left[\begin{array}{c}
y_{C A, \hat{u}}^{\hat{1}} \\
\vdots \\
y_{C A, \hat{u}}^{\hat{n}_{s}} \\
y_{D L 1, \hat{u}}^{\hat{1}} \\
\left(y_{D L 1, \hat{u}}^{\hat{2}}\right) \\
\vdots \\
y_{D L 1, \hat{u}}^{\hat{n}_{s}}
\end{array}\right]=\left[\begin{array}{cccc}
G_{\hat{u}}^{\hat{1}} & \mathbf{0} & 1 & 0 \\
& \vdots & & \\
G_{\hat{u}}^{\hat{n}_{s}} & \mathbf{0} & 1 & 0 \\
\mathbf{0} & G_{\hat{\hat{1}}}^{\hat{1}} & 0 & 1 \\
(\mathbf{0}) & \left(G_{\hat{u}}^{\hat{2}}\right) & (0) & (1) \\
\vdots & & \\
\mathbf{0} & G_{\hat{u}}^{\hat{n}_{s}} & 0 & 1
\end{array}\right]\left[\begin{array}{c}
u \\
\dot{u} \\
c \delta t_{u} \\
c \dot{\delta} t_{u}
\end{array}\right]+v
$$

In the case of Doppler bias estimation, if the Doppler bias is detected in the $j$-th satellite, then Doppler bias can be added as unknown parameter $b_{D L 1}^{j}$ for the $j$-th component of $y_{D L 1, \hat{u}}^{\hat{s}}$ to the state vector $\eta_{L}$, and the related column is added to the last column of observation matrix. The initial values of the unknown parameters $b_{D L 1}^{j}$ are set to the difference values between Doppler range-rates and code delta-ranges. And then the system noise related to the parameters are added into covariance matrix $Q(t)$ in (49).

For example, if $j=2$, the unknown parameter for $2_{n d}$ Doppler observable and the related column with brackets are added into the state vector and the observation matrix, respectively as follows.

$\left[\begin{array}{c}y_{C A, \hat{u}}^{\hat{1}} \\ \vdots \\ y_{C A, \hat{u}}^{\hat{n}_{s}} \\ y_{D L 1, \hat{u}}^{\hat{1}} \\ y_{D L 1, \hat{u}}^{\hat{2}} \\ \vdots \\ y_{D L 1, \hat{u}}^{\hat{n}_{s}}\end{array}\right]=\left[\begin{array}{ccccc}G_{\hat{u}}^{\hat{1}} & \mathbf{0} & 1 & 0 & (0) \\ & \vdots & & & \\ G_{\hat{u}}^{\hat{n}_{s}} & \mathbf{0} & 1 & 0 & (0) \\ \mathbf{0} & G_{\hat{\hat{u}}}^{\hat{1}} & 0 & 1 & (0) \\ \mathbf{0} & G_{\hat{u}}^{2} & 0 & 1 & (1) \\ & \vdots & & & \\ \mathbf{0} & G_{\hat{u}}^{\hat{n}_{s}} & 0 & 1 & (0)\end{array}\right]\left[\begin{array}{c}u \\ \dot{u} \\ c \delta t_{u} \\ c \dot{\delta} t_{u} \\ \left(b_{D L 1}^{2}\right)\end{array}\right]+v$

\section{Experimental results}

The experiment of Kalman filter-based positioning simulation has been carried out to detect and correct the Doppler biases. Table 2 shows the simulation conditions.

Table 2: Simulation conditions

\begin{tabular}{|l||c|c|c|}
\hline & $\begin{array}{c}\text { No } \\
\text { correction }\end{array}$ & $\begin{array}{c}\text { Doppler } \\
\text { bias } \\
\text { exclusion }\end{array}$ & $\begin{array}{c}\text { Doppler } \\
\text { bias } \\
\text { estimation }\end{array}$ \\
\hline \hline C/A noise variance $\left[\mathrm{m}^{2}\right]$ & $5^{2}$ & $5^{2}$ & $5^{2}$ \\
\hline $\begin{array}{l}\text { Doppler noise variance } \\
{\left[(\mathrm{m} / \mathrm{s})^{2}\right]}\end{array}$ & $0.5^{2}$ & $0.5^{2}$ & $0.5^{2}$ \\
\hline $\begin{array}{l}\text { System noise variance } \\
\text { for Doppler bias } b_{D L 1}^{j}\end{array}$ & - & - & 0.01 \\
\hline $\begin{array}{l}\text { Percent point for } \\
\chi^{2} \text { tests }\end{array}$ & - & $\chi_{0.05}^{2}(1)$ & $\chi_{0.05}^{2}(1)$ \\
\end{tabular}

The statistical tests of the innovation processes for excluding the biased Doppler shifts, or estimating the Doppler biases can be done at every epoch.

In the case of Doppler bias exclusion, if Doppler shifts would include biases in one epoch, all target components of $y_{D L 1, \hat{u}}^{\hat{s}}$ whose innovation value surpass the percent point of $\chi^{2}$ test are excluded. Dynamically judging if the target components are excluded or not is comparatively a simpler method, however it can detect spike fluctuations of Doppler innovation values. In the case of Doppler bias estimation, when accepting $H_{1}$ by $\chi^{2}$ test continue for three epochs, Doppler estimation processes for the target satellites are started. The continuous 


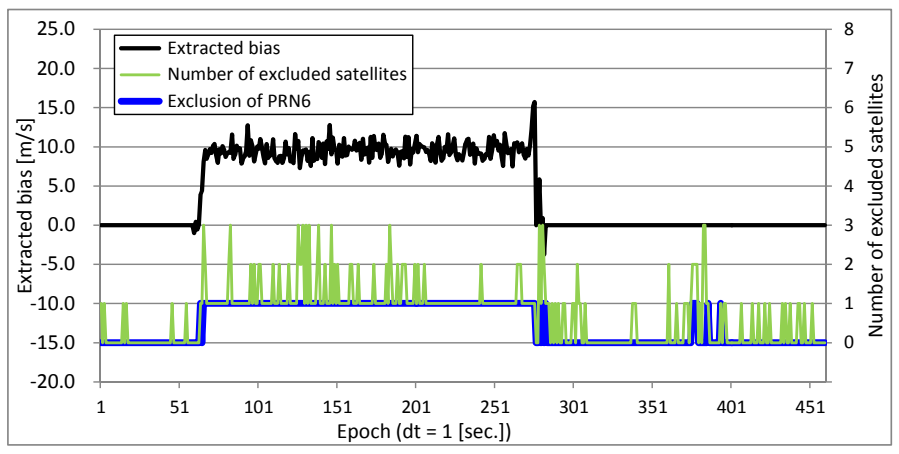

Fig. 5: Doppler bias exclusion

three epoch detections can mask spikes of Doppler innovation values. Although the $\chi^{2}$ test detection is done based on the single observation epoch and Doppler observable, the proposed method can be easily extended to the detection based on multiple epochs or observables. When the Doppler bias is left, the estimated value by the unknown parameter $b_{D L 1}^{j}$ is approaching to zero. If the value would be less than the specific value, the estimation process is stopped.

In the case of Doppler bias exclusion, during the bias period, the Doppler observable of the target satellite PRN6 which includes the simulation bias is synchronously excluded by the bias exclusion method (see Fig. 5). However in the 30 percent of the bias period, a few of other satellites are excluded together with PRN6. The number of Doppler shift observables which are utilized for positioning are decreased. In the case of Doppler bias estimation, during the bias period, the bias estimation for target satellite PRN6 which includes the bias starts after three epochs at the start point of the period, and finish with several epoch delays at the end point of the period (see Fig. 6). The estimated bias values are almost the same as the bias values. The Doppler biases of other satellites are estimated, the bias estimations are immediately stopped after several epochs when the estimation value is less than specific threshold level.

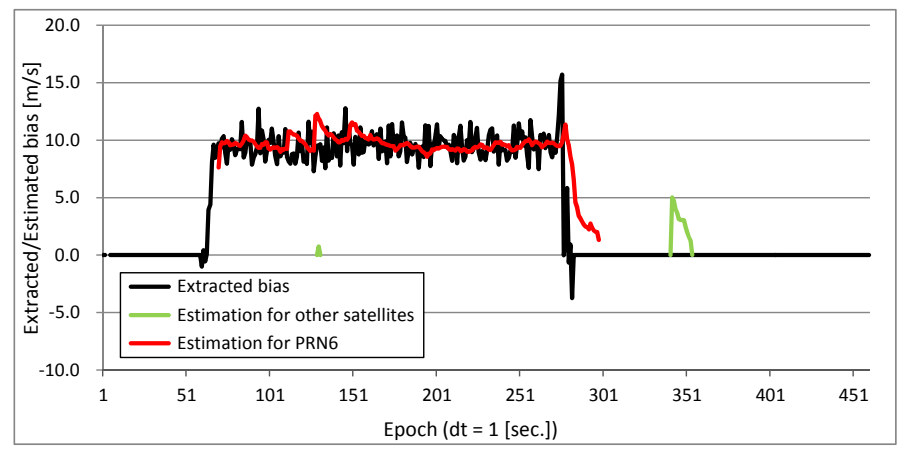

Fig. 6: Doppler bias estimation
Fig. 7 shows the comparison of the positioning results with and without the Doppler bias correction. The trajectories corrected by the Doppler bias exclusion or estimation are improved. They are almost the same positioning as the normal positioning without the Doppler bias.

Fig.8 shows ENU errors of correction results. The positioning results by original log data which include no Doppler bias are regarded as the reference trajectory for evaluating positioning errors. As for no correction, the ENU errors are more than 20 [m] in the period of Doppler bias. Contrarily, the ENU errors of Doppler bias exclusion or estimation corrections are almost less than $20[\mathrm{~m}]$. As for Doppler bias estimation, the errors are bigger at the start and end points of the Doppler bias period, however the Doppler bias estimation can provide more accurate positioning results than

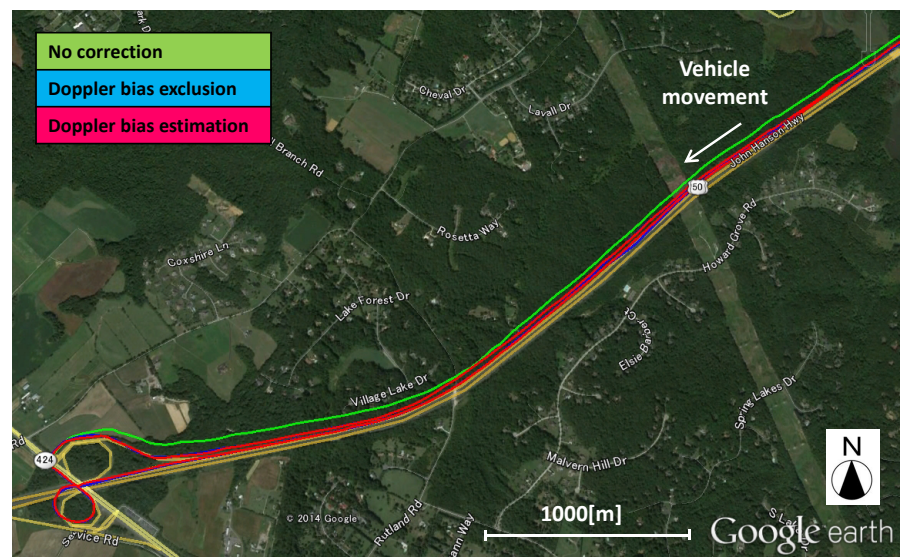

Fig. 7: Correction results

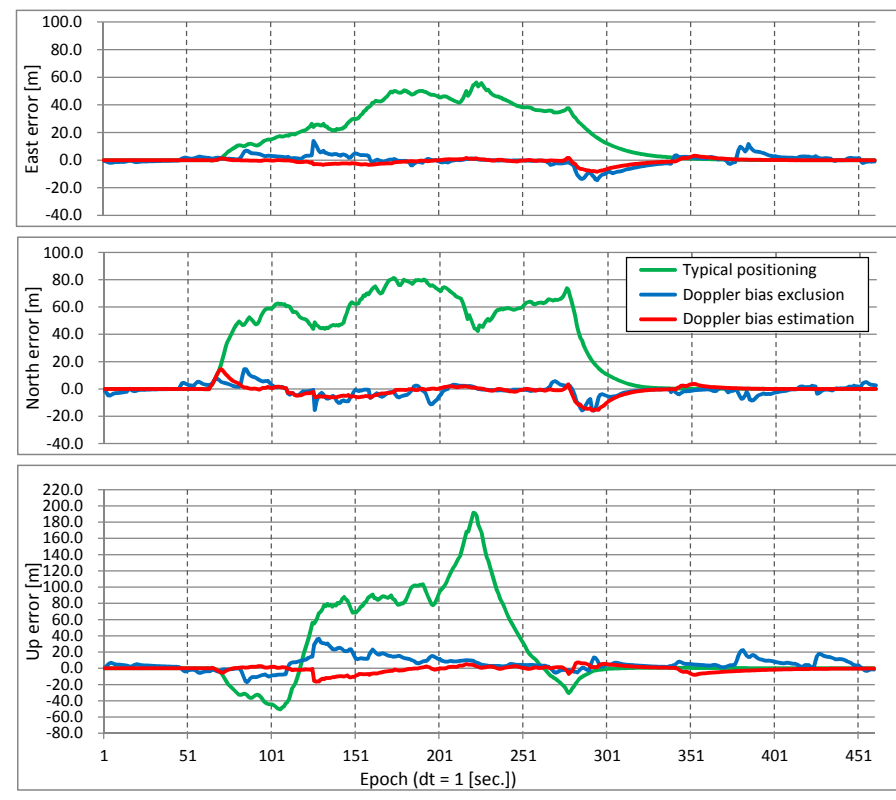

Fig. 8: ENU errors for correction 
the Doppler bias exclusion. As for Doppler bias exclusion, the other satellites' Doppler observables except for PRN6's are excluded for the positioning, that is supposed to cause more inaccurate positioning.

\section{Conclusions}

In this paper, GPS Kalman filter-based positioning and an extracted Doppler bias sample are used to simulate the positioning error, and the error has been reproduced under open sky environment. The typical noise variance for $\mathrm{C} / \mathrm{A}$ code and Doppler shift observables are defined to $5^{2}\left[\mathrm{~m}^{2}\right]$ and $0.5^{2}\left[(\mathrm{~m} / \mathrm{s})^{2}\right]$, respectively. Doppler frequency shift observables are more reliable than $\mathrm{C} / \mathrm{A}$ code pseudoranges, however when Doppler shift observables have bias errors, the biases cause positioning errors even under open sky environments. $\chi^{2}$ tests based on the normalized innovation process of Kalman filter is utilized for detecting the biased Doppler shift observables. And Doppler bias exclusion or estimation methods are utilized to remove the impacts of Doppler biases and correct the positioning shift errors. The Doppler bias exclusion method is slightly affected by the spike noises of Doppler observables, on the other hand the bias estimation method masks the noises by starting the estimation after three epoch detections. The both methods almost can remove the continuous bias impacts, and correct the positioning errors under open sky environments.

\section{REFERENCES}

[1] S. Sugimoto and Y. Kubo: Unified Methods of Point and Relative Positioning Based on GNSS Regression Equations, Proc. 19th Int. Tech. Meeting of the Satellite Division of The Institute of Navigation (ION GNSS 2006), pp. 345-358, Fort Worth, Texas, Sep, (2006).

[2] S. Sugimoto and R. Shibasaki (Eds.): GPS Handbook (in Japanese), Asakura-Shoten, Tokyo (2010).

[3] Y. Kubo, K. Sone and S. Sugimoto: Cycle Slip Detection and Correction for Kinematic GPS Based on Statistical Test of Innovation Processes, Proc. of ION GNSS 17th Int. Tech. Meeting of the Satellite Div., pp. 1438-1447, Long Beach, CA, 21-24 Sept. (2004).

[4] M. Kamimura, R. Tomita, Y. Kubo and S. Sugimoto: Detection of Cycle Slips and Multipath in GNSS RTK Precise Point Positioning, Proc. 24th Int. Technical Meeting of the Satellite Division of the Institute of Navigation, Portland OR (Sept. 2011).

[5] M. Bahrami and M. Ziebart: Doppler-Aided Positioning: Improving Single-Frequency RTK in the Urban Environment, GPS World, 22(5):47-56, May 2011.

[6] M. Bahrami and M. Ziebart: A Kalman Filterbased Doppler-smoothing of Code Pseudoranges in GNSS-Challenged Environments, Proc. 24th Int. Technical Meeting of the Satellite Division of the Institute of Navigation, Portland OR (Sept. 2011).

[7] A. Chabata, Y. Suzuki, Y. Kubo and S. Sugimoto: RTK-PPP Algorithms using GNSS Observables from Few Satellits, 25th Int. Technical Meeting of the Satellite Division of the Institute of Navigation, Nashville TN (Sept. 17-21, 2012).

[8] B. W. Parkinson and J. J. Spilker Jr. (Eds.): Global Positioning System: Theory and Applications, Vol. I, II, AIAA (1997).

[9] Y. Kubo, K. Sone and S. Sugimoto: Fault Detection in Carrier Phase GPS Positioning Based on Hypotheses Testing of Innovation Processes, International Journal of Innovative Computing, Information and Control Volume 1, Number 3, pp461478, September 2005.

[10] R. A. Singer: Estimating Optimal Tracking Filter Performance for Manned Maneuvering Targets, IEEE Trans. Aerospace and Electronic Systems, Vol. AES-6, No. 4, pp. 473-483 (1970).

[11] T. Aoki, Y. Shimogaki, T. Ikki, M. Tanikawara, S. Sugimoto, Y. Kubo and K. Fujimoto: Cycle Slip Detection in Kinematic GPS with a Jerk Model for Land Vehicles, Int. J. of Innovative Computing, Information and Control, vol. 5, no. 1, pp. 153-166, January (2009).

[12] T. Aoki and S. Sugimoto: Dynamical Models for Automobile Movements, Int. J. of Innovative Computing, Information and Control, vol. 6, no. 1, pp. 3-14, January (2009)

[13] C. Uratani, K. Sone, Y. Muto, S. Maruo, and S. Sugimoto: A New Method for Carrier-PhaseBased Precise Point, Proc. 16th Int. Technical Meeting of the Satellite Division of the Institute of Navigation (ION GPS/GNSS 2003), pp. 809-818, Portland, OR, Sept. (2003).

[14] Y. Muto, Y. Kubo, Y. Kubo and S. Sugimoto: New Dynamical Models for Kinematic GPS Positioning, Proc. 17th Int. Technical Meeting of the Satellite Division of the Institute of Navigation (ION GNSS 2004), Long Beach, CA, Sept. (2004).

[15] P. Misra and P. Enge: Global Positioning System -Signals, Measurements, and Performance, 2nd Edition, Ganga-Jamuna Press, Massachusetts (2006). 